BMJ Open Sport \& Exercise Medicine

\section{Effects of a novel exercise training protocol of Wingate-based sprint bouts dispersed over a day on selected cardiometabolic health markers in sedentary females: a pilot study}

To cite: Ho BH, Lim I, Tian R, et al. Effects of a novel exercise training protocol of Wingate-based sprint bouts dispersed over a day on selected cardiometabolic health markers in sedentary females: a pilot study. BMJ Open Sport \& Exercise Medicine 2018:4:e000349. doi:10.1136/ bmjsem-2018-000349

Accepted 23 May 2018
Check for updates

${ }^{1}$ Changi Sports Medicine Centre, Changi General Hospital, Singapore, Singapore

${ }^{2}$ Sport Science and Medicine, Singapore Sport Institute, Sport Singapore, Singapore, Singapore ${ }^{3}$ Department of Physiology, Yong Loo Lin School of Medicine, National University of Singapore, Singapore, Singapore

Correspondence to Dr Boon Hor Ho; wenhu17@ gmail.com

\section{ABSTRACT}

Purpose Sprint interval training (SIT) provides a strong stimulus for improving cardiovascular fitness, which is among the key markers for premature mortality. Recent literature demonstrated that SIT protocols with as few as two stacked $20 \mathrm{~s}$ Wingate Anaerobic Test (WAnT) cycle sprints provide sufficient training stimulus for a robust increase in maximal aerobic power. However, this effect is lost when only one bout is performed. This suggests training adaptation is still dependent on the volume of SIT. Therefore, the purpose of this study was to determine the effects of three dispersed $30 \mathrm{~s}$ WAnT bouts, done over a day but interspersed with 4 hours of recovery time, on selected cardiometabolic health markers.

Methods Eighteen sedentary women, age $36 \pm 8$ years, were recruited and underwent 8 weeks of supervised training using the WAnT protocol, 3 days a week. Criterion measure of cardiovascular fitness (ie, $\mathrm{VO}_{2 \text { peak }}$ ), skinfolds and blood lipids such as triglyceride, low density lipoprotein (LDL) and high density lipoprotein (HDL) were measured before and after training intervention.

Results $\mathrm{VO}_{2}$ improved by a mean of $14.0 \%$ after training $(21.7 \pm 5.7$ vs $24.7 \pm 5.7 \mathrm{~mL} / \mathrm{kg} / \mathrm{min}, \mathrm{p}<0.01)$. No significant change was observed for body fat and lipid profile.

Conclusion Performing three dispersed WAnT bouts with a 4-hour recovery period between bouts throughout a day, 3 days per week for 8 weeks provides sufficient training stimulus for a robust increase in $\mathrm{VO}_{2 \text { peak }}$, which is comparable with other previous SIT protocols with very short recovery intervals. However, no other changes in the other cardiometabolic health markers were detected.

\section{INTRODUCTION}

Moderate to high intensity continuous or aerobic-type exercises have consistently been promoted and emphasised by the statutory authorities as the main modalities of regular physical activity for improving cardiovascular fitness. However, receptivity to these exercise recommendations remains poor, most likely due to the substantial time commitment associated with these exercise protocols.

\section{What are the new findings?}

Very short but maximal intensity exercises, even with single bouts dispersed over the day, can still provide sufficient training stimulus for robust increase in $\mathrm{VO}_{2}$

How might it impact on clinical practice in the future?

Considering one of the common barriers for the poo adherence to exercise routine is the lack of time, this novel 'dispersed' exercise protocol is clearly a time-efficient 'office-tractive' exercise protocol that may be prescribed as alternative to the conventional manner in which sprint interval trainings are currently being conducted.

Consequently, sedentary behaviour and inactivity remains a serious healthcare issue. ${ }^{1}$ To address the common barrier of lack of time to exercise, ${ }^{2}$ supramaximal intensity or sprint interval training (SIT) has been proposed as a time-efficient alternative exercise strategy. ${ }^{3}$ These SIT protocols are generally characterised by repeated sessions of short exercise, typically at intensities that elicit $\geq 85 \%$ of the individual's maximal oxygen uptake and interspersed with short periods of rest or low-intensity effort for recovery. ${ }^{4}$ SIT protocols can induce physiological remodelling similar to continuous moderate intensity training, despite reduced time commitment and a relatively small total exercise volume. ${ }^{5-7}$ However, the low volume of SIT does not necessarily imply a shorter time commitment for exercise, as the need for recovery periods between repetitions can result in a total training time commitment of more than 30 min per session (see table 1 on comparison of previous SIT based studies). 


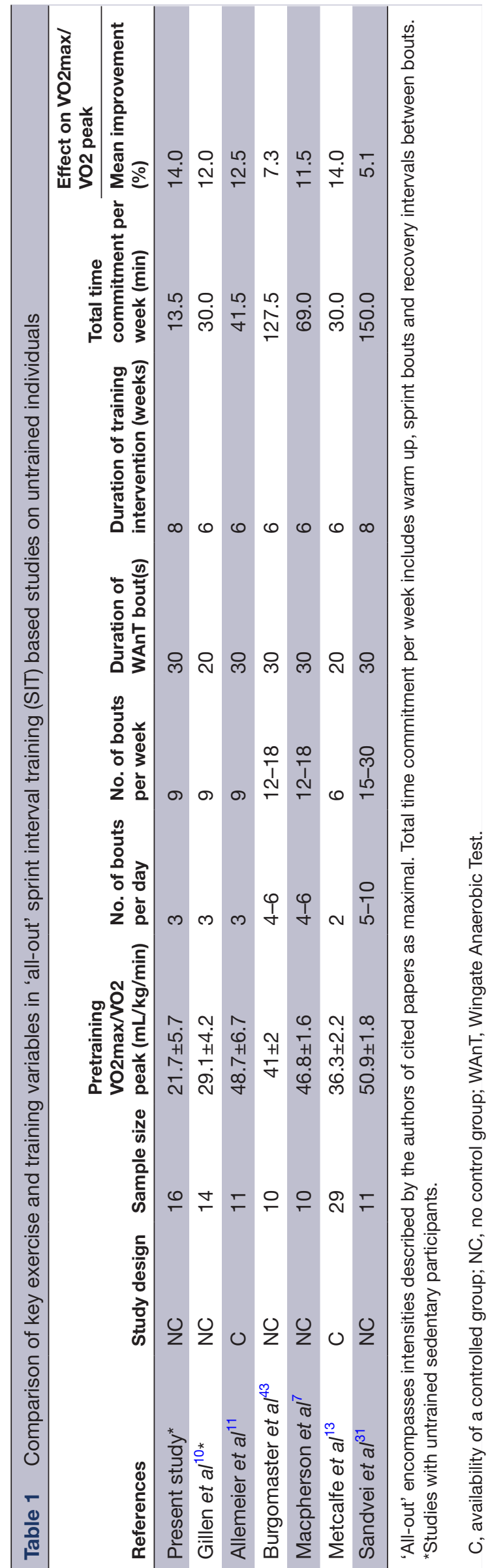

The mechanisms by which SIT exercise improves maximal oxygen uptake are still poorly understood, and it remains unknown how the training stimulus can be optimised to achieve increase in maximal oxygen uptake, using the smallest amount of time and effort. Recent evidence suggests that SIT protocols with fewer and shorter intervals $^{8-12}$ were reported to increase maximal oxygen uptake, similar to traditional SIT protocols. Notably, Metcalfe and colleagues ${ }^{13}$ demonstrated in their study that a mean increase of $14 \%$ can be achieved by performing just two 20 $\mathrm{s}$ all-out sprints in an exercise day, performed 3 days a week, for 6 weeks. Recently, Songsorn and colleagues ${ }^{14}$ pushed the boundaries further by piloting a new protocol with a single $20 \mathrm{~s}$ sprint once a day, performed three times a week, over 4 weeks. However, this strategy did not yield a significant improvement in maximal oxygen uptake likely due to the low daily training stimulus associated with the short length of the SIT protocol.

The purpose of the present pilot study therefore was to determine whether a novel low-volume SIT exercise can overcome the limitations of current SIT protocols. Specifically, we examined the impact of a training protocol that involved three dispersed $30 \mathrm{~s}$ Wingate Anaerobic Test (WAnT)-based cycle bouts performed over the day; each bout within a $1.5 \mathrm{~min}$ (60 s warm-up and $30 \mathrm{~s}$ exercise) time commitment block. A dispersed sprint protocol was selected as WAnT has been shown to provide the highest power output and accumulated work when done as a single bout. ${ }^{415}$ Therefore, the rationale was that by dispersing the three single sprint bouts over a typical day, each bout was more likely to be better tolerated in the untrained population as the WAnT bouts separated by a prolonged rest period and were not clustered as typically seen in traditional interval training. The rest period in between the sprints was set at 4 hours to allow full recovery from each WAnT bout ${ }^{16}$ and to coincide with the break periods of office workers to allow for practical adoption of this novel exercise protocol, that is, exercise can be performed at the start of the working day, during lunch time and at the end of the work day. It should be noted that the $2 \times 20 \mathrm{~s}$ SIT study by Metcalfe and colleagues ${ }^{13}$ and other recent studies were completed as a stacked cluster ('all out' bouts completed in a single session). To our knowledge, no study has endeavoured to investigate the training-induced effects of a single bout of WAnT performed several times throughout the day, that is, a dispersed protocol, with several hours of passive rest time between bouts. Hence, this pilot study sought to determine whether this novel protocol approach to performing the SIT could improve selected cardiometabolic health markers, including body mass, body fat, maximal oxygen uptake and blood lipids, in a group of sedentary females.

\section{METHODS}

\section{Participants}

Eighteen untrained, female participants (mean $\pm \mathrm{SD}$; age $36 \pm 8$ year, body mass index (BMI) $25.2 \pm 4.0 \mathrm{~kg} / \mathrm{m}^{2}$, $\mathrm{VO}_{2 \text { peak }} 21.6 \pm 5.4 \mathrm{~mL} / \mathrm{kg} / \mathrm{min}$ ) were recruited. Participants were staff from a local hospital who were habitually 
sedentary (due to prolonged sitting) more than 8 hours a day due to the nature of their work. ${ }^{17}$ None of them had been involved in any systematic or regular exercise for the previous 12 months. Participation inclusion criteria were: non-habitually exercising individuals with involvement in low to moderate intensity physical activities $<75$ min per week and presenting with no contraindications to exercise as determined by a standard physical activity readiness questionnaire. ${ }^{18}$ All individuals were medically screened by a physician prior to the commencement of the study and were certified healthy with no chronic health issues. Furthermore, all subjects were informed about how lifestyle changes may potentially influence the outcome of the study and were instructed to maintain their normal diet and levels of physical activity (apart from the current training programme) throughout the period of the study. The aim and requirements of the study were fully explained to all participants in written and verbal form before they were asked to provide signed consent.

\section{Experimental protocol}

The study's experimental protocol consists of (1) baseline testing on an electronically braked cycle ergometer and blood measures and also familiarisation with the air-braked cycle ergometer; (2) an 8-week WAnT bouts dispersal protocol training intervention; and (3) posttesting measures as per baseline testing.

\section{Baseline measures}

Baseline testing for all participants consisted of measurements of body mass, skinfolds (as a proxy for levels of body fat), maximal oxygen uptake (or $\mathrm{VO}_{2 \text { peak }}$ ) on a cycle ergometer and fasting blood lipids. Specifically, on separate occasions, all participants performed: (1) a ramp exercise orientation on a cycle ergometer (VIAsprint 150P, Vyaire Medical, Germany) to determine maximal aerobic capacity and (2) several 3-10 s of maximal and submaximal exertion practices on a cycle ergometer (Wattbike Trainer, Nottingham, UK) to familiarise the participants with the WAnT exercise. These were all completed 1 week before commencement of the 8-week training.

\section{Body mass and skinfolds}

Individuals' body mass was measured using standardised instruments (Avamech B1000, Singapore). The individuals' skinfold thickness (at seven sites) ${ }^{19}$ were measured using Harpenden skinfold callipers, and all measures (pretraining and post-training for all individuals) were made by the same trained anthropometrist.

\section{Maximal oxygen uptake cycle test $\left(\mathrm{VO}_{2 \text { peak }}\right)$}

One week before the start of the training programme, participants performed a continuous incremental ramp cycling test to exhaustion on an electronically-braked cycle ergometer (VIAsprint 150P, Germany) to determine maximal aerobic capacity 1 week before the start of the training programme.
Participants were asked not to perform strenuous exercise or consume caffeine or alcohol the day before the test and to drink $500 \mathrm{~mL}$ of water the morning of the testing day to ensure sufficient hydration. Participants completed a 2 min warm-up at $50 \mathrm{~W}$ after which the cycle ergometer resistance was increased by $1 \mathrm{~W}$ every $3 \mathrm{~s}$ until volitional exhaustion despite strong verbal encouragement. Oxygen uptake $\left(\mathrm{VO}_{2}\right)$ was measured continuously throughout the test using a calibrated metabolic cart (MasterScreen CPX, Germany) to determine maximal aerobic capacity. $\mathrm{VO}_{2 \text { peak }}$ was defined as the highest 30 $\mathrm{s}$ of oxygen uptake value recorded during the cycle test. In all of the tests, two or more of the following criteria were met: respiratory exchange ratio (RER) $>1.15$, heart rate within 10 beats of age-predicted maximum (207$0.7 \times$ age) and/or the individual showed symptoms of volitional exhaustion. The post-intervention maximal aerobic capacity test was performed 1 week after the final training session as per pretraining procedures.

\section{Blood markers}

On a separate day, with each participant fasted for at least 8 hours the night before, approximately $5 \mathrm{~mL}$ of blood were collected using the BD Vacutainer blood collection system in the hospital and sent directly to the hospital biochemical laboratory. Triglyceride, low density lipoprotein (LDL)-cholesterol and high density lipoprotein (HDL)-cholesterol analyses were performed on a modular analyser (Roche Cobas 8000, Switzerland). The intra-assay coefficient of variation (CV) for blood lipids were all within $5 \%$.

\section{Exercise training intervention}

Participants performed an 8-week WAnT bouts dispersal protocol training programme consisting of three non-consecutive exercise days per week, for a total of 72 bouts. On each exercise day, participants performed three bouts of WAnT, with each of the WAnT bout separated by 4 hours. All exercise was conducted on an air-braked cycle ergometer (Wattbike Trainer, Nottingham, UK). Each WAnT bout was preceded by $60 \mathrm{~s}$ of warm-up cycling at intensity of less than $50 \mathrm{~W}$ resistance and followed by a single 'allout' 30 s cycle sprint effort. Participants were asked to cycle at 20-30 revolutions per min during the warm-up and towards the end of this $60 \mathrm{~s}$ duration, a countdown of ' $3,2,1$ and Go' was provided where the individual was instructed to exert maximally at 'Go' and then to try to sustain their cadence throughout the $30 \mathrm{~s}$ exercise duration. Participants received strong verbal encouragement throughout the exercise duration. On completion of the $30 \mathrm{~s}$ of exercise, individuals slowed down gradually and stopped within $10 \mathrm{~s}$ or less. At this time, ratings of perceived exertion (RPEs; Borg's categorical 6-20 scale) were administered to the individuals. Thereafter, individuals dismount from the ergometer and returned back to their work station or go back to perform their daily routine. The sprint bouts were scheduled 4 hours apart, that is, 'dispersed' throughout the day, to fit into 
the work schedule of the participants. The bouts were typically performed between 08:00 and 09:00 (before starting work), between 13:00 and 14:00 (lunch break) and between 17:00 and 18:00 (after work). Because of the challenging nature of SIT, participants performed a modified $20 \mathrm{~s}$ WAnT bout during the initial training weeks 1 and 2 to allow them to adapt to the exercise intensity. The $30 \mathrm{~s}$ protocol was then used for the remaining 6 weeks of training. Absolute mean power output (MPO) was recorded during the $30 \mathrm{~s}$ training sessions as provided by the cycle ergometer's performance monitor. Relative mean power is calculated by taking into account the individual's body mass measured at the start of every session. All training sessions were conducted and supervised by a trained exercise physiologist. Participants used the same Wattbike ergometer throughout their 8 weeks of training.

There are two ways to set the resistance of a Wattbike cycle ergometer. One is modulating the air-brake damper, and the other involves increasing the magnetic resistance of the flywheel. The air-damper can be set from level 1 to level 10 (higher means more air is allowed into the front air-vent implying a greater resistance when cycling) and magnetic resistance (levels 1-7; a higher setting implies a greater inertia akin to cycling up a steep slope). During the familiarisation to the WAnT exercise, each individual was allowed on a trial-and-error basis and with assistance from our experienced laboratory trainer to set her own individualised air-brake and magnetic resistance settings. These two resistance setting values were duly recorded for each individual and used throughout her 8 weeks of training sessions. Progression in each individual's training stimuli was ensured with each individual exerting their own perceived maximal effort during each of the training session, that is, increasing in cycle cadence where the faster and harder the individual exerts, the greater the generated power output of the cycle. ${ }^{20}$ Thus, training progression is a 'natural' process whereby as the individual gets 'fitter', the faster and harder she exerts during the $30 \mathrm{~s}$ cycle, the greater will be her power output generated during exercise.

To be included in the final data analysis, participants had to complete at least $75 \%$ of the training sessions. ${ }^{21}$ A second $\mathrm{VO}_{2 \text { peak }}$ test, fasting lipids and other measurements described above were obtained 1 week after the final training session. Participants were asked to maintain their usual physical activity behaviour and their normal dietary intake for the duration of the study. ${ }^{22}$

\section{Classification of training-induced response}

The CV for the measurement of $\mathrm{VO}_{2 \text { peak }}$ in our laboratory is determined to be at $2.3 \%$ (our own laboratory's unpublished data). However, a $5 \% \mathrm{CV}$ for $\mathrm{VO}_{2 \text { peak }}$ was adopted in the present study as a larger variability was expected as the present participants were all untrained. All the participants were classified according to their specific response to the 8-week exercise training intervention. ${ }^{23}$ A responder was defined as an individual who demonstrated an increase that was greater than two times the $\mathrm{CV}$ of $\mathrm{VO}_{2 \text { peak }}$. Conversely, an adverse responder was defined as an individual who demonstrated a decrease that was greater than two times the CV from zero, and a non-responder was defined as an individual who failed to demonstrate an increase or decrease that was greater than two times the CV away from zero. ${ }^{23}$

\section{Statistical analysis}

Categorical data were presented in frequency and percentage. Numeric data were presented as mean \pm SD. Significance differences between pretraining and posttraining in primary outcome, $\mathrm{VO}_{2}$ peak and secondary outcomes, such as MPO and blood markers, were determined using paired sample t-test. A two-tailed $\mathrm{p}$ value of $<0.05$ was considered statistically significant. Statistical analysis was performed with SPSS statistical software, V.19.0.

\section{RESULTS}

Of the 18 participants, 17 of them completed at least $75 \%$ of the training sessions (mean training sessions compliance was $88.6 \% \pm 8.8 \%$ ). With regards to $\mathrm{VO}_{2 \text { peak }}$, there were no outliers as assessed by inspection of a boxplot for values greater than 1.5 box-lengths. However, out of the 17 participants, 1 participant was removed from final analysis due to unusual results. The participant had an $>80 \%$ increase in her $\mathrm{VO}_{2 \text { peak }}$, which is abnormally high for typical physiological adaptation from a low-volume SIT protocol; hence the results are only for 16 subjects' data.

The MPO averaged across the three WAnT bouts performed during the day was significantly lower in the first training day in week 3 compared with the last training day in training week $8(\mathrm{n}=16 ; 4.32 \pm 0.72$ vs $4.65 \pm 1.06 \mathrm{~W} /$ $\mathrm{kg}, \mathrm{p}<0.01)$, which clearly indicate training-induced improvement in power output of the participants towards the end of the training programme. The mean weekly RPE from training week 1 to week 8 was: $16.9 \pm 2.1$, $16.6 \pm 2.5,17.8 \pm 1.9,17.8 \pm 2.0,18.3 \pm 1.7,18.4 \pm 1.5,18.5 \pm 1.4$ and 18.3 \pm 1.4 , respectively $(F$ ratio $=2.43, \mathrm{p}=0.023)$. Post hoc analysis, however, indicated no significant difference between mean RPEs measured during each of the eight training weeks (all comparisons $\mathrm{p}>0.05$ ). Importantly, the dispersed WAnT bout training protocol showed an increase in mean $\mathrm{VO}_{2 \text { peak }}$ by $14.0 \%$ (pretraining: $21.7 \pm 5.7$ $\mathrm{mL} / \mathrm{kg} / \mathrm{min}$ vs post-training: $24.7 \pm 5.7 \mathrm{~mL} / \mathrm{kg} / \mathrm{min}$, $\mathrm{p}<0.01 ; \mathrm{n}=16$; figure 1$)$. The difference in $\mathrm{VO}_{2 \text { peak }}$ was normally distributed, as assessed by Shapiro-Wilk's test $(p=0.425)$. There was no significant difference in the $\mathrm{HR}_{\text {max }}$ measured during the maximal aerobic power cycle tests (pretraining: $167 \pm 15$ beats/min vs post-training: $171 \pm 16$ beats $\cdot \min , \mathrm{p}>0.05 ; \mathrm{n}=16$ ).

There was also no significant difference in MPO between taken from the first WAnT bout of the day (performed between 08:00 and 09:00) and the last bout (performed between 17:00 and 18:00) of the exercise day during training week $3(\mathrm{n}=16$; bout $1: 4.37 \pm 0.73$ vs bout 3: $4.27 \pm 0.80 \mathrm{~W} / \mathrm{kg}, \mathrm{p}>0.05)$ and during training week 


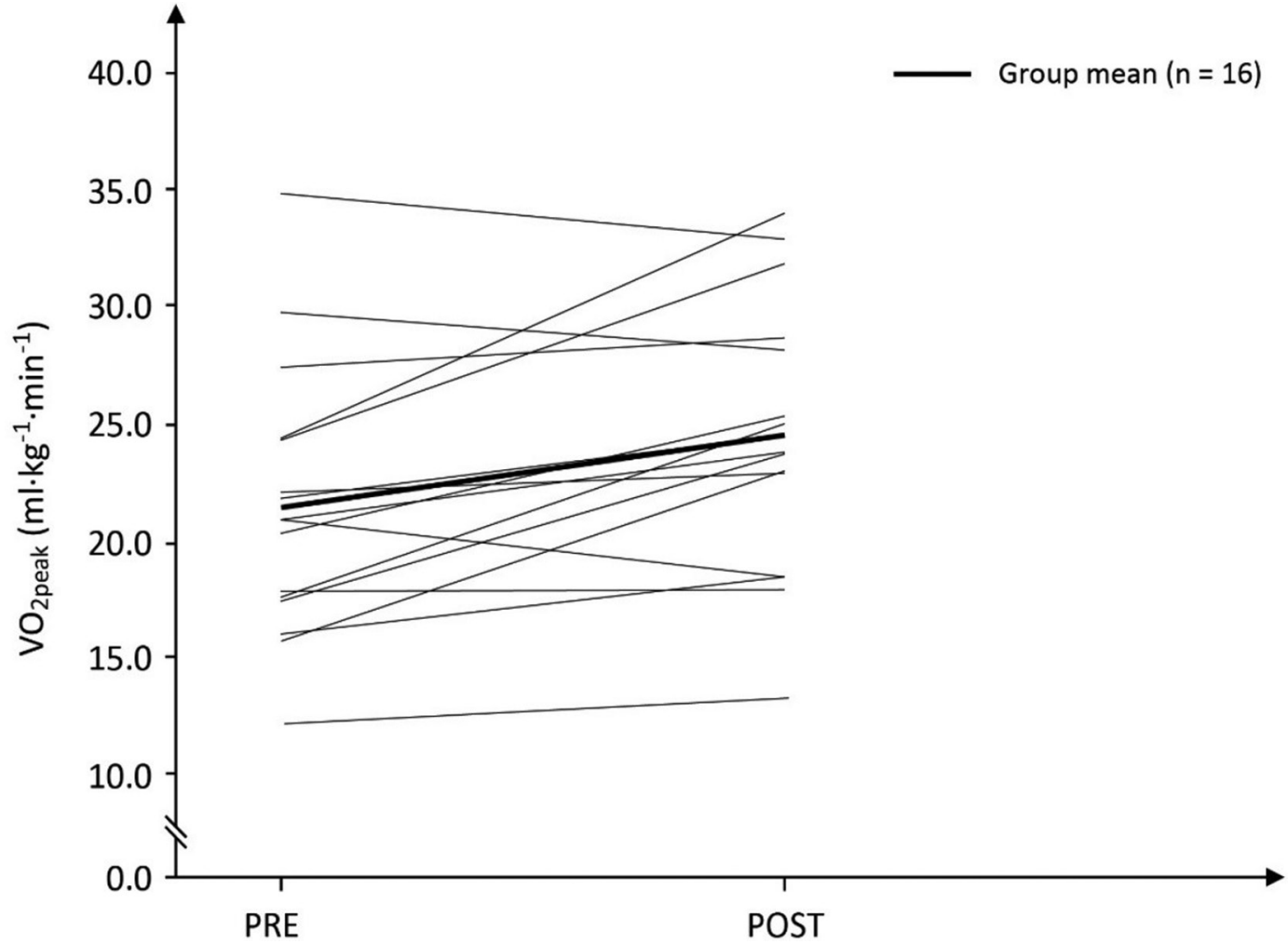

figure 1 Effects of the novel dispersed Wingate bouts training protocol on cardiovascular fitness.

$8(\mathrm{n}=16$; bout $1: 4.70 \pm 1.06$ vs bout $3: 4.70 \pm 1.09 \mathrm{~W} / \mathrm{kg}$, $\mathrm{p}>0.05)$.

Body mass and skinfold measures were not significantly different before compared to after training (all $\mathrm{p}>0.05$, table 2). Similarly, resting blood lipids profile also showed no significant changes from pretraining to post-training (all p $>0.05$, table 2 ).

\section{DISCUSSION}

The results of the present study demonstrated that an 8-week novel exercise intervention consisting of very brief but maximal efforts, dispersed over the day, is associated with improved cardiovascular fitness (or $\mathrm{VO}_{2 \text { peak }}$ ) in females. This beneficial effect occurred, however, independently from changes in body mass and selected cardiometabolic health markers during the training period. Nonetheless, given that maximal aerobic power has been shown to be one of the strongest prognostic markers for cardiovascular health and premature death, ${ }^{13}$ the results indicate the potency of the new

Table 2 Markers of health outcomes measured in the study $(n=16)$

\begin{tabular}{lcc}
\hline & Pretraining & Post-training \\
\hline Body mass (kg) & $61.7 \pm 10.2$ & $61.6 \pm 10.5$ \\
Skinfolds (mm) & $156 \pm 34$ & $167 \pm 35$ \\
HDL (mmol/L) & $1.73 \pm 0.39$ & $1.73 \pm 0.37$ \\
LDL (mmol/L) & $2.77 \pm 0.73$ & $2.85 \pm 0.65$ \\
Triglyceride (mmol/L) & $0.95 \pm 0.52$ & $0.89 \pm 0.39$ \\
\hline
\end{tabular}

dispersed SIT exercise protocol, and more importantly, the cardiovascular fitness improvement was observed despite the time commitment of a mere 1.5 min per day of exercise time (excluding warm-up), for 3 days a week (see table 1).

The underlying mechanism(s) responsible for the observed improvement in $\mathrm{VO}_{2 \text { peak }}$ cannot be determined with the design of the present study. Nonetheless, previous reviews, however, have suggested that the enhanced cardiovascular fitness is likely due to enhanced central adaptations such as increases in stroke volume or blood volume $^{25}$ and/or the result of peripheral adaptation of skeletal muscles by upregulating various mitochondrial enzymes activities such as citrate synthase, pyruvate dehydrogenase, 3-hydroxyacy CoA dehydrogenase and cytochrome oxidase ${ }^{2627}$ and increasing mitochondria density. ${ }^{10} 28$ It has been suggested that increases in the central and peripheral adaptations were due to the very rapid glycogen depletion associated with the WAnT exercise. ${ }^{29}$ When the WAnT bouts are performed in the typical stack clustered protocol, only the first WAnT bout is fuelled predominantly by muscle glycogen with the subsequent bouts involving a greater contribution of fats oxidation. ${ }^{15}$ Therefore, in the present dispersed protocol, it may be argued that each bout of the WAnT over the day was performed with the highest usage of muscle glycogen during each $30 \mathrm{~s}$ effort causing a relatively greater muscle glycogen depletion over the three bouts.

The findings of the present study extend the previous literature of SIT's efficacy ${ }^{10}$ by showing that the sprint repetitions can be separated over long durations, yet still 
maintaining the positive effects. One possible reason for the sustained training-induced effects is that when the WAnT bouts are separated by long periods of rest or recovery, or performed in a dispersed manner as in the present study, the exercise stimulus of each bout is maximised. This is supported by the finding that there was no significant difference in MPO of WAnT bout 1 (ie, performed early in the morning) relative to the MPO of WAnT bout 3 (performed in the late afternoon) observed during training week 3 and week 8 . Indeed, this implies that each of the WAnT performed at three times of the day would have resulted in the maximal activation of the metabolic pathways or maximum increases in production of specific metabolites or higher signalling impulses between different kinases, transcriptional regulatory proteins and cells relative to a situation in which the three WAnT bouts were performed in a clustered fashion which likely lead to the enhanced training-induced adaptations within the sedentary females.

Considering the commonly reported barrier of lack of available time for exercise,$^{2}$ there is also a need to design an exercise protocol that provides adequate fitness and health benefits and enhances motivation to perform exercise by overcoming key barriers. One possible alternative strategy could be to define the minimum volume of maximal sprint exercise required to improve both fitness and health indices whilst also increasing exercise adherence. To date, the exercise protocol utilised in the current study represents the smallest volume of exercise (when using total training time per week), relative to recent studies, that has been shown to induce positive effects on peak oxygen uptake, albeit in sedentary, low-level of fitness individuals (see table 1 for comparisons).

There is a dearth of sufficient evidence in current literature to indicate that a larger number of sprint repetitions or a greater total volume of sprint exercise will lead to superior cardiovascular or metabolic adaptations. Metcalfe and colleagues ${ }^{13}$ showed that very low volumes of supramaximal exercise $-2 \times 20 \mathrm{~s}$ exercise session-are associated with improvements in $\mathrm{VO}_{2 \max }$ typically seen with much higher volumes of sprints. ${ }^{4}{ }^{3}$ Recent studies ${ }^{1013}$ have demonstrated that the SIT exercise repetitions may be as low as $2-3$ bouts of $20 \mathrm{~s}$ per day to provide a positive stimulus, however, when reduced by Songsorn and colleagues ${ }^{14}$ to just a single bout of $20 \mathrm{~s}$ per day, performed three times per week on alternate days, this positive effect was lost. We hypothesised that there would be a significant deficit of stimulus if these $30 \mathrm{~s}$ bouts are performed only once per exercise day, especially when compared with a clustered $3 \times 30 \mathrm{~s}$ WAnT protocol bouts. Therefore, we maintained the number of bouts as previous studies ${ }^{10}{ }^{13}$ but eliminated the need for recovery time between bouts by integrating an extended 4-hour recovery interval period as part of the individual work day. This 4-hour duration provided full recovery from each bout and therefore, allowed our participants to undergo three sprint bouts per exercise day without appreciable accumulated fatigue.
It should be noted that the absolute number of bouts performed per day in the present study is similar to the number of bouts per day employed in recent SIT protocols (table 1). Hence, these two key strategies: (1) completion of a greater absolute workload for the second and third Wingate bouts of the day through dispersion and (2) increasing the daily volume of SIT exercise with the 4-hour resting period which allowed 3 maximal sprint bouts per day of exercise, are likely responsible for the positive change in $\mathrm{VO}_{\text {2peak }}$ observed in the present study.

Blood lipids are well-established metabolic risk factors that are associated with cardiovascular diseases. ${ }^{32}{ }^{33}$ The present study's dispersed WAnT bouts protocol showed no impact on blood lipids, which was similarly observed in other traditional or typical SIT protocols. For example, six sessions of 4-6 WAnT bouts (with 4.5 min recovery between bouts) performed over a 2-week period showed no change in blood lipids profile in sedentary overweight, obese men. ${ }^{34}$ Similarly, repeated cycle efforts of $8 \mathrm{~s}$ with $12 \mathrm{~s}$ recovery over a 20 -min period, performed 4 days a week for 5 weeks, did not show any significant change in the blood lipid profile of obese females. ${ }^{35}$ However, the intensity of cycling in the latter study was estimated to be $\sim 80 \%$ of $\mathrm{VO}_{2 \max }$ and not of supramaximal. Interestingly, a recent study showed improvement in LDL but not in HDL and total cholesterol; however, the study's SIT consisted of four clustered $20 \mathrm{~s}$ sprint at $175 \% \mathrm{VO}_{2 \max }$, three times per week for 12 weeks. ${ }^{36}$ The combined findings of these studies suggest that a positive change in lipid profiles is dependent on a dose-response of energy expenditure, exercise duration and/or volume of exercise. ${ }^{35}$ Thus, the present study with its low volume exercise profile was unlikely to exert any positive effects on the individuals' lipids profile.

High-intensity interval training has been shown to be effective in reducing body fat in overweight females. ${ }^{37}$ In the present study, however, there was no significant change in body mass and skinfolds measurements of the participants after 8 weeks of SIT training. The positive effects for body fat reduction were not achieved even though most of the participants were overweight females (mean BMI: $24.9 \pm 4.1 \mathrm{~kg} / \mathrm{m}^{2}$, based on Asian population). However, there is substantial difference in the mean BMI between participants in the study of Mirghani and Yousefi ${ }^{37}$ and those in the present study $\left(29.5 \pm 3.5\right.$ vs $24.9 \pm 4.1 \mathrm{~kg} / \mathrm{m}^{2}$, respectively). Based on current weight loss concepts, individuals with high initial weight typically have faster initial weight loss due to higher caloric expenditure for similar intensity and duration of exercises and increased ease in initial diet caloric reduction. This is supported by the findings of Nackers $e t a l,{ }^{38}$ who demonstrated that individuals with higher BMIs have higher initial rates of weight loss. Moreover, our exercise protocol was relatively short with 8 weeks of training, compared with the usual training duration of 6 months or more, to achieve effective weight loss. These differences in initial weight and BMIs, in addition to the shorter exercise period and exercise volume of the present exercise protocol, may 
have resulted in a much slower rate of fat and weight loss. Another potential reason could be the low energy expenditure of the current dispersed exercise protocol. When SIT bouts are performed in a clustered fashion, the last few bouts are fuelled primarily by fat oxidation rather than via anaerobic glycolysis ${ }^{15}$ with greater levels of excess post-exercise oxygen consumption per exercise session, which can lead to a higher overall fat loss. Thus, it is very likely that the present dispersed protocol resulted in each WAnT bout utilising primarily glycogen as opposed to fats. This, in addition to the likelihood of lower excess post-exercise oxygen consumption for the entire day's exercise may have led to the low body fat loss in our female subjects, although direct investigation is required to confirm our assertion.

Even though the present study dispersed exercise protocol provides a sufficient training stimulus for a robust increase in $\mathrm{VO}_{2 \text { peak }}$, it did not reduce the incidence of non-response, similar to previously reported study. ${ }^{39}$ The present study reports an overall rate of $37.5 \%$ non-responders for $\mathrm{VO}_{2 \text { peak }}$ across all participants studied (6 out of 16 participants), with a single adverse responder. It was suggested that the incidence of non-response following SIT is likely due to individual variability in adaptive response to the training stimulus. ${ }^{4041}$ Recently, however, Montero and Lundby ${ }^{42}$ showed the abolishment of all non-response to exercise training in individuals who trained an additional 240 min per week for 6 weeks, after they initially showed no training-induced adaptations after exercising between $60 \mathrm{~min}$ and $300 \mathrm{~min}$ per week for 6 weeks. Therefore, it is possible that a low training stimulus or insufficient training effort could be a significant factor resulting in the non-response, rather than individual genetic variation to exercise adaptation. ${ }^{42}$ While the exact dose of SIT required for more effective training-induced adaptations remain unclear and likely to be subjected to individual variability, it would appear that increasing the number of training days per week from 3 to perhaps 4-5 days is an important consideration, at least in the case of the present 'dispersed' WAnT bouts exercise protocol.

Our study is not free of limitations. Dietary intake outside the training intervention was not monitored. The present study employed a repeated measure design with no control group, which may introduce bias. However, many previous studies have already validated the positive effects of SIT on maximal aerobic power, and recent studies conducted were similarly conducted without a control group as in the present study (see table 1). Finally, the present study provides information on exercise training and cardiorespiratory fitness within a small sedentary female population $(n=16)$, and therefore, there is clearly a need to conduct larger population-based studies to determine the effectiveness of this dispersing SIT protocol. Nonetheless, a major advantage of the present study's novel 'dispersal' protocol for performing the WAnT bouts is that it is ideal for the practical 'real world' purpose of fitting the exercise easily into the daily schedule of an individual office worker, and concurrently, using his or her work time in between the bouts as a natural 'built-in' recovery period. With this new strategy, exercise duration was effectively shortened to $1.5 \mathrm{~min}$ per day (3 bouts of $30 \mathrm{~s}$ of exercise time) or a total time commitment of only 13.5 min per week (including warm-up).

\section{CONCLUSION}

The present study showed that $3 \times 30 \mathrm{~s}$ WAnT bouts performed throughout the day, conducted for 3 non-consecutive days per week for 8 weeks, improved peak oxygen uptake in sedentary females. The protocol employed in the present study involved a training time commitment of a mere 4.5 min per day (including warm-up) that was considerably lower than previous WAnT-based SIT studies and provides further evidence of the potential for very brief, intense bouts of exercise to elicit improvements in cardiovascular fitness (ie, a key cardiometabolic health indicator) in a time efficient manner. This suggests that this novel SIT 'dispersed' exercise protocol may offer a genuinely time-efficient alternative to the conventional SIT cardiorespiratory exercise training.

Acknowledgements The authors would like to thank and acknowledge the exercise physiologists in Changi Sports Medicine Centre who made this study possible. Much appreciation to Associate Professor Mike Newton for his inputs on the final version of the manuscript.

Funding This study was funded by Changi General Hospital, Singapore (grant number: CHF2015-21-P).

Competing interests None declared.

Patient consent Obtained.

Provenance and peer review Not commissioned; externally peer reviewed.

Open access This is an Open Access article distributed in accordance with the Creative Commons Attribution Non Commercial (CC BY-NC 4.0) license, which permits others to distribute, remix, adapt, build upon this work non-commercially, and license their derivative works on different terms, provided the original work is properly cited and the use is non-commercial. See: http://creativecommons.org/ licenses/by-nc/4.0/

(C) Article author(s) (or their employer(s) unless otherwise stated in the text of the article) 2018. All rights reserved. No commercial use is permitted unless otherwise expressly granted.

\section{REFERENCES}

1. Hawari NS, Al-Shayji I, Wilson J, et al. Frequency of breaks in sedentary time and postprandial metabolic responses. Med Sci Sports Exerc 2016;48:2495-502.

2. Korkiakangas EE, Alahuhta MA, Laitinen JH. Barriers to regular exercise among adults at high risk or diagnosed with type 2 diabetes: a systematic review. Health Promot Int 2009;24:416-27.

3. Gillen JB, Gibala MJ. Is high-intensity interval training a time-efficient exercise strategy to improve health and fitness? Appl Physiol Nutr Metab 2014;39:409-12.

4. Weston M, Taylor KL, Batterham AM, et al. Effects of low-volume high-intensity interval training (HIT) on fitness in adults: a metaanalysis of controlled and non-controlled trials. Sports Med 2014;44:1005-17.

5. Gibala MJ. Physiological adaptations to low-volume high-intensity interval training. Sports Science Exchange 2015;28:1-6.

6. Gibala MJ, McGee SL. Metabolic adaptations to short-term highintensity interval training. Exerc Sport Sci Rev 2008;36:58-63.

7. Macpherson RE, Hazell TJ, Olver TD, et al. Run sprint interval training improves aerobic performance but not maximal cardiac output. Med Sci Sports Exerc 2011;43:115-22.

8. Hazell TJ, Macpherson RE, Gravelle BM, et al. 10 or 30-s sprint interval training bouts enhance both aerobic and anaerobic performance. Eur J Appl Physiol 2010;110:153-60. 
9. Zelt JG, Hankinson PB, Foster WS, et al. Reducing the volume of sprint interval training does not diminish maximal and submaximal performance gains in healthy men. Eur J Appl Physiol 2014;114:2427-36.

10. Gillen JB, Percival ME, Skelly LE, et al. Three minutes of all-out intermittent exercise per week increases skeletal muscle oxidative capacity and improves cardiometabolic health. PLoS One 2014;9:e111489.

11. Allemeier CA, Fry AC, Johnson $P$, et al. Effects of sprint cycle training on human skeletal muscle. J Appl Physiol 1994;77:2385-90.

12. Ijichi $\mathrm{T}$, Hasegawa $\mathrm{Y}$, Morishima $\mathrm{T}$, et al. Effect of sprint training: training once daily versus twice every second day. Eur J Sport Sci 2015;15:143-50.

13. Metcalfe RS, Babraj JA, Fawkner SG, et al. Towards the minimal amount of exercise for improving metabolic health: beneficial effects of reduced-exertion high-intensity interval training. Eur J Appl Physiol 2012;112:2767-75.

14. Songsorn $\mathrm{P}$, Lambeth-Mansell A, Mair JL, et al. Exercise training comprising of single 20-s cycle sprints does not provide a sufficient stimulus for improving maximal aerobic capacity in sedentary individuals. Eur J Appl Physiol 2016;116:1511-7.

15. McCartney N, Spriet LL, Heigenhauser GJ, et al. Muscle power and metabolism in maximal intermittent exercise. J Appl Physiol 1986;60:1164-9.

16. Larsen I, Welde B, Martins C, et al. High- and moderate-intensity aerobic exercise and excess post-exercise oxygen consumption in men with metabolic syndrome. Scand J Med Sci Sports 2014;24:e174-e179.

17. Tremblay M. Sedentary Behaviour Research Network. Letter to the editor: standardized use of the terms "sedentary" and "sedentary behaviours". Appl Physiol Nutr Metab 2012;37:540-2.

18. Thomas S, Reading J, Shephard RJ. Revision of the physical activity readiness questionnaire (PAR-Q). Can J Sport Sci 1992;17:338-45.

19. Jackson AS, Pollock ML. Generalized equations for predicting body density of men. Br J Nutr 1978;40:497.

20. Driller MW, Argus CK, Shing CM. The reliability of a 30-s sprint test on the Wattbike cycle ergometer. Int J Sports Physiol Perform 2013;8:379-83.

21. Neufer PD. The effect of detraining and reduced training on the physiological adaptations to aerobic exercise training. Sports Med 1989;8:302-21.

22. Kavaliauskas M, Steer TP, Babraj JA. Cardiorespiratory fitness and aerobic performance adaptations to a 4-week sprint interval training in young healthy untrained females. Sport Sci Health 2017;13:17-23.

23. Edgett BA, Bonafiglia JT, Raleigh JP, et al. Reproducibility of peak oxygen consumption and the impact of test variability on classification of individual training responses in young recreationally active adults. Clin Physiol Funct Imaging 2017.

24. Myers J, Prakash M, Froelicher V, et al. Exercise capacity and mortality among men referred for exercise testing. $N$ Engl J Med 2002;11346:33-4.

25. Esfandiari S, Sasson Z, Goodman JM. Short-term high-intensity interval and continuous moderate-intensity training improve maximal aerobic power and diastolic filling during exercise. Eur J Appl Physiol 2014;114:331-43.

26. Gist NH, Fedewa MV, Dishman RK, et al. Sprint interval training effects on aerobic capacity: a systematic review and meta-analysis. Sports Med 2014;44:269-79.
27. Vollaard NBJ, Metcalfe RS, Williams S. Effect of number of sprints in an SIT session on change in V'O2max: a meta-analysis. Med Sci Sports Exerc 2017;49:1147-56.

28. Nalcakan GR. The effects of sprint interval vs. continuous endurance training on physiological and metabolic adaptations in young healthy adults. J Hum Kinet 2014;44:97-109.

29. Metcalfe RS, Koumanov F, Ruffino JS, et al. Physiological and molecular responses to an acute bout of reduced-exertion high-intensity interval training (REHIT). Eur J Appl Physiol 2015;115:2321-34

30. Milanović Z, Sporiš G, Weston M. Effectiveness of high-intensity interval training (HIT) and continuous endurance training for VO2max improvements: a systematic review and meta-analysis of controlled trials. Sports Med 2015;45:1469-81.

31. Sandvei M, Jeppesen PB, Støen L, et al. Sprint interval running increases insulin sensitivity in young healthy subjects. Arch Physiol Biochem 2012;118:139-47.

32. Blair SN, Kohl HW, Barlow CE, et al. Changes in physical fitness and all-cause mortality. A prospective study of healthy and unhealthy men. JAMA 1995;273:1093-8.

33. Lee DC, Sui X, Artero EG, et al. Long-term effects of changes in cardiorespiratory fitness and body mass index on all-cause and cardiovascular disease mortality in men: the aerobics center longitudinal study. Circulation 2011;124:2483-90.

34. Whyte LJ, Gill JM, Cathcart AJ. Effect of 2 weeks of sprint interval training on health-related outcomes in sedentary overweight/obese men. Metabolism 2010;59:1421-8.

35. Kong Z, Fan X, Sun S, et al. Comparison of high-intensity interval training and moderate-to-vigorous continuous training for cardiometabolic health and exercise enjoyment in obese young women: a randomized controlled trial. PLoS One 2016;11:e0158589.

36. Bagley L, Slevin M, Bradburn S, et al. Sex differences in the effects of 12 weeks sprint interval training on body fat mass and the rates of fatty acid oxidation and $\mathrm{VO}_{2 \max }$ during exercise. BMJ Open Sport Exerc Med 2016;2:e000056.

37. Mirghani SJ, Yousefi MS. The effect of interval recovery periods during HIIT on liver enzymes and lipid profile in overweight women. Sci Sports 2015;30:147-54.

38. Nackers LM, Ross KM, Perri MG. The association between rate of initial weight loss and long-term success in obesity treatment: does slow and steady win the race? Int J Behav Med 2010;17:161-7.

39. Smith-Ryan AE, Melvin MN, Wingfield HL. High-intensity interval training: modulating interval duration in overweight/obese men. Phys Sportsmed 2015;43:107-13.

40. Gurd BJ, Giles MD, Bonafiglia JT, et al. Incidence of nonresponse and individual patterns of response following sprint interval training. Appl Physiol Nutr Metab 2016;41:229-34.

41. Bonafiglia JT, Rotundo MP, Whittall JP, et al. Inter-individual variability in the adaptive responses to endurance and sprint interval training: a randomized crossover study. PLoS One 2016;11:e0167790.

42. Montero D, Lundby C. Refuting the myth of non-response to exercise training: 'non-responders' do respond to higher dose of training. J Physiol 2017;595:3377-87.

43. Burgomaster KA, Howarth KR, Phillips SM, et al. Similar metabolic adaptations during exercise after low volume sprint interval and traditional endurance training in humans. J Physiol 2008;586:151-60. 